\title{
Differential effects of dairy snacks on appetite ratings, but not overall energy intake
}

\author{
A. Dougkas ${ }^{1,3}$, A. M. Minihane ${ }^{2}$, D. I. Givens ${ }^{3}$, P. Yaqoob ${ }^{1}$ and C. K. Reynolds ${ }^{3}$ \\ ${ }^{1}$ Hugh Sinclair Human Nutrition Group, School of Chemistry, Food Biosciences and Pharmacy, Faculty of Life Sciences \\ University of Reading, Reading RG6 6AP, UK, ${ }^{2}$ Department of Nutrition, Norwich Medical School, University of East \\ Anglia (UEA), Norwich NR4 7TJ, UK and ${ }^{3}$ School of Agriculture, Policy and Development, Faculty of Life Sciences, \\ University of Reading, Reading RG6 6AR, UK
}

Excess body weight has many causes, with dietary factors and physical activity levels being the most important at a population level ${ }^{(1)}$. Dietary regulation of appetite may contribute to the prevention and management of excess body weight ${ }^{(2)}$. There is a paucity of available evidence detailing the effects of increased dairy product consumption on appetite and energy intake ${ }^{(3)}$. The aim of the present study was to examine the effect of consumption of individual dairy products as snacks on appetite and subsequent ad libitum lunch energy intake.

In a randomised crossover trial, forty overweight men [age: 32 (SD 9) years; BMI ( $\mathrm{kg} / \mathrm{m}^{2}$ ): 27 (SD 2)] attended four sessions one week apart and received three isoenergetic $(830 \mathrm{~kJ})$ and isovolumetric $(410 \mathrm{ml})$ servings of dairy snacks or water (control) $120 \mathrm{~min}$ after breakfast. Subjective appetite ratings (hunger, desire to eat, fullness and prospective consumption) were determined using visual analogue scales (VAS) throughout the morning, and ad libitum energy intake as a lunch-time meal was assessed 90 min after the intake of snacks.
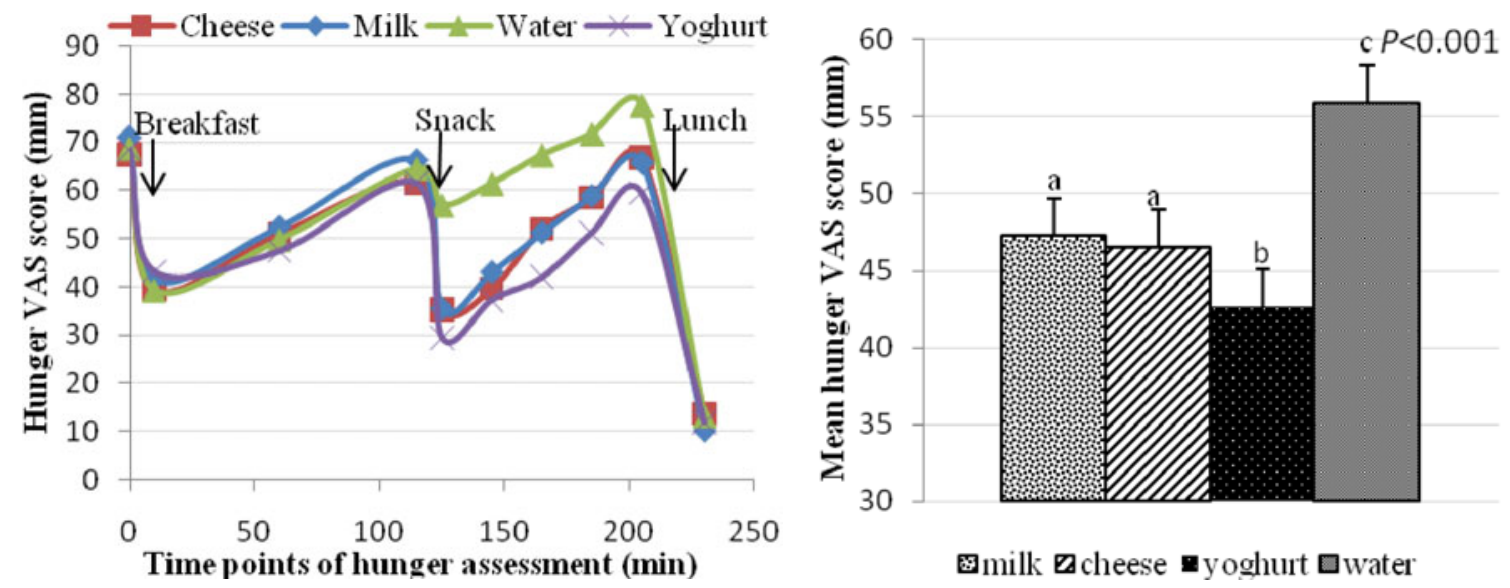

Fig. 1. Hunger ratings throughout the morning.

Results showed that among the milk products, yoghurt had the greatest suppressive effect on appetite. Hunger rating was 8,10 and $24 \%$ $(P<0.001)$ lower after intake of yoghurt compared with intake of cheese, milk and water, respectively (Fig. 1). Similar results were observed for ratings of desire to eat and prospective consumption $(P<0.01$ and $P<0.05$, respectively). Fullness rating was $9 \%(P=0.004)$ and $30 \%(P<0.001)$ higher after intake of yoghurt compared with intake of milk and water, respectively, although there was no difference relative to cheese $(P=0.186)$. However, these appetite ratings between dairy products were not associated with reduced energy intake at the subsequent lunch. Energy intake was 119 and $12 \%(P<0.02)$ lower after intake of yoghurt, cheese and milk, respectively compared with water [4312 (SE 226) kJ]. In conclusion, yoghurt had the greatest effect on suppressing subjective appetite ratings, but did not affect subsequent food intake.

\footnotetext{
1. Abete I, Astrup A, Martinez JA et al. (2010) Nutr Rev 68, 214-231.

2. Rolls ET (2007) Obesity Rev 8, 67-72.

3. Teegarden D \& Gunther CW (2008) Nutr Rev 66, 601-605.
} 